\title{
Probability Functions of Order Statistics from Discrete Uniform Distribution
}

\author{
A. Metin Karakaş ${ }^{1, *}$ and S. Çalik ${ }^{2}$ \\ ${ }^{1}$ Department of Statistics, Bitlis Eren University \\ 13000 Bitlis, Turkey \\ ${ }^{2}$ Department of Statistics, Firat University \\ 23119 Elaziğ, Turkey \\ *Corresponding author's email: aysekarakas5767 [AT] gmail.com
}

\begin{abstract}
In this paper, we firstly give basic definitions and theorems for order statistics. Later, we show that $r$. probability function of order statistics from discrete uniform distribution can be obtained in another form.
\end{abstract}

Keywords--- Order statistics, Distribution function, Discrete Uniform Distribution, Moments, Probability Function.

\section{INTRODUCTION}

In literature, there are many papers about order statistics. [1] gave systematic account of some theory of ordered statistics for discrete case. [2] studied order statistics of discrete distribution. [3] gave simple expression for the joint probability function and applied some of our results to theory of order statistics for the discrete uniform distribution. [4] mentioned applications, characterization, approaches for the statistical properties of order statistics of discrete distribution. [5] studied on Markov property bearing structure and mentioned conditional Markov property of order statistics of discrete distribution. [6] were given the distribution of the sample range of discrete order statistics. [7] found the first two moments of discrete order statistics with different method. [8] gave algebraic expression values using probability function of sample maximum from discrete order statistics. [9] obtained sample extreme moments of discrete uniform distribution order statistics. [10] obtained $\mathrm{m}$. moments of discrete uniform distribution order statistics. [11] found given m. moments of sample extreme from discrete uniform distribution order statistics. In this study the $r$. probability function of order statistics from discrete uniform distribution is obtained. [12] obtained mth raw moments of sample extremes of order statistics from discrete uniform distribution and given numerical values are shown in table form.

\section{METERIAL AND METHOD}

\subsection{Order Statistics}

Let $X_{1}, X_{2}, \ldots, X_{n}$ be random variables possibly dependent a necessarily identically distributed. By rearranging them in no decreasing order of magnitude, we obtain order statistics $X_{1: n} \leq X_{2: n} \leq, \ldots, \leq X_{n: n}$. Thus, $X_{1: n}=$ min $\left(X_{1}, X_{2}, \ldots, X_{n}\right), X_{2: n}$ the second smallest observation among $X_{1}, X_{2}, \ldots, X_{n}$ and finally $X_{1: n}=\max$ $\left(X_{1}, X_{2}, \ldots, X_{n}\right)$. Under stronger assumption that $X_{1}, X_{2}, \ldots, X_{n}$ are independent and identically distributed random variables with an arbitrary cumulative distribution function $F$, we obtain that the cdf of $X_{r: n}$;

$$
\begin{aligned}
& F_{r: n}(x)=\operatorname{Pr}\left(X_{r: n} \leq x\right)=\operatorname{Pr}\left(X_{1}, X_{2}, \ldots, X_{n} \text { at least r rv's among } X_{1}, X_{2}, \ldots, X_{n} \leq x\right) \\
&=\sum_{i=r}^{n} \operatorname{Pr}\left(X_{1}, X_{2}, \ldots, X_{n} \text { exactly j rv's among } X_{1}, X_{2}, \ldots, X_{n}\right. \\
&=\sum_{i=r}^{n}\left(\begin{array}{c}
n \\
i
\end{array}\right)[F(x)]^{i}[1-F(x)]^{n-i},-\infty<x<\infty .
\end{aligned}
$$

Furthermore, using the following equation

$$
\sum_{i=r}^{n}\left(\begin{array}{c}
n \\
i
\end{array}\right) p^{i}(1-p)^{n-i}=\int_{0}^{p} \frac{n !}{(r-1) !(n-r) !} t^{r-1}(1-t)^{n-r} d t, \quad 0<p<1
$$


the cdf of $X_{r: n}$

$$
\begin{gathered}
F_{r: n}(x)=\int_{0}^{F(x)} \frac{n !}{(r-1) !(n-r) !} t^{r-1}(1-t)^{n-r} d t \\
=I_{F(x)}(r, n-r+1),-\infty<x<\infty
\end{gathered}
$$

can be written as. In this place, $I$ is incomplete beta function. This expression of $F_{r: n}(x)$ want to get discrete or continuous any main mass is provided for. In discrete distribution, for probability mass function of $X_{r: n}$ there are three approaches.

\section{Approach 1 (Binomiaol Count)}

For each possible value of $X_{r: n}$

$$
f_{r: n}(x)=F_{r: n}(x)-F_{r: n}(x-) \text {. }
$$

Therefore,

$$
f_{r: n}(x)=\sum_{i=r}^{n}\left(\begin{array}{c}
n \\
i
\end{array}\right)\left\{[F(x)]^{i}[1-F(x)]^{n-i}-[F(x-)]^{i}[1-F(x-)]^{n-i}\right\}
$$

is written.

\section{Approach 2 (Beta Integral Form)}

From (2.3) and (2.4), using expression of $F_{r: n}(x)$, probability mass function of $X_{r: n}$ is written;

$$
f_{r: n}(x)=\int_{F(x-)}^{F(x)} \frac{n !}{(r-1) !(n-r) !} t^{r-1}(1-t)^{n-r} d t .
$$

\section{Approach 3 (Multiple Argument)}

For an observation value $X$, let us consider the following three different events; $\{X<x\},\{X=x\},\{X>x\}$ ,respectively. The probability of this events are $F(x-), F(x)$ and $1-F(x) .\left\{X_{r: n}=x\right\}$ event can occur in $r(n-r+1)$ different ways. $i=0,1, \ldots, r-1$ and $s=0,1, \ldots, n-r$ including $(r-1-i)$ units observation value is less than $\mathrm{x},(n-r-s)$ observation value is greater than $\mathrm{x}$ and in the remaining equal to $\mathrm{x}$. Then it can be written

$$
f_{r: n}(x)=\sum_{i=0}^{r-1} \sum_{s=0}^{n-r} \frac{n ![F(x-)]^{r-1-i}[1-F(x)]^{n-r-s}[f(x)]^{s+i+1}}{(r-1-i) !(n-r-s) !(s+i+1) !}
$$

Here, if $x=0$, then $F(x-)=0$.

\section{RESULTS}

Let probability mass function be $f(x)=1 / k$ and cumulative distribution function be $F(x)=x / k, x=1,2, \ldots, k$ of $X_{1}, X_{2}, \ldots, X_{n}$ which are $\mathrm{n}$ unit independent and identically distributed random variables. From ( 2.1), cumulative distribution function of $X_{r: n}$;

$$
F_{r: n}(x)=\sum_{i=r}^{n}\left(\begin{array}{l}
n \\
i
\end{array}\right)\left(\frac{x}{k}\right)^{i}\left(1-\frac{x}{k}\right)^{n-i}, \quad x=1,2, \ldots, k
$$

and from (2.6), probability mass function of $X_{r: n}$ can be written as

Theorem

$$
\begin{aligned}
f_{r: n}(x) & =\int_{F(x-)}^{F(x)} C(r: n) u^{r-1}(1-u)^{n-r} d u \\
& =\int_{(x-1) / k}^{x / k} C(r: n) u^{r-1}(1-u)^{n-r} d u
\end{aligned}
$$


Let $X_{1}, X_{2}, \ldots, X_{n}$ be random sample of size $\mathrm{n}$ from discrete uniform distribution and $X_{r: n}$ is $\mathrm{r}$ th order statistics. Probability function of $X_{r \cdot n}$;

$$
f_{r: n}(x)=\sum_{i=1}^{r} \frac{n !}{(i-1) !(n-i+1) !}\left[\left(\frac{x-1}{k}\right)^{i-1}\left(\frac{k-x+1}{k}\right)^{n-i+1}-\left(\frac{x}{k}\right)^{i-1}\left(\frac{k-x}{k}\right)^{n-i+1}\right]
$$

and

$$
f_{r: n}(x)=\sum_{i=r}^{n} \frac{n !}{i !(n-i) !}\left[\left(\frac{x}{k}\right)^{i}\left(\frac{k-x}{k}\right)^{n-i}-\left(\frac{x-1}{k}\right)^{i}\left(\frac{k-x+1}{k}\right)^{n-i}\right]
$$

can be found with any of expression.

\section{Proof}

In (3.2), if we integrate for $r=1$,

$$
f_{1: n}(x)=\left(\frac{k-x+1}{k}\right)^{n}-\left(\frac{k-x}{k}\right)^{n}
$$

if we integrate for $r=2$,

$$
f_{2: n}(x)=n\left[\left(\frac{x-1}{k}\right)\left(\frac{k-x+1}{k}\right)^{n-1}-\left(\frac{x}{k}\right)\left(\frac{k-x}{k}\right)^{n-1}\right]+f_{1: n}(x)
$$

if we integrate for $r=3$,

$$
f_{3: n}(x)=\frac{n(n-1)}{2 !}\left[\left(\frac{x-1}{k}\right)^{2}\left(\frac{k-x+1}{k}\right)^{n-2}-\left(\frac{x}{k}\right)^{2}\left(\frac{k-x}{k}\right)^{n-2}\right]+f_{2: n}(x)
$$

is obtained. Therefore, we conclude that

$$
f_{r: n}(x)=\frac{n !}{(r-1) !(n-r+1) !}\left[\left(\frac{x-1}{k}\right)^{r-1}\left(\frac{k-x+1}{k}\right)^{n-r+1}-\left(\frac{x}{k}\right)^{r-1}\left(\frac{k-x}{k}\right)^{n-r+1}\right]+f_{r-1: n}(x) .
$$

From (3.3), (3.4), (3.5) and (3.6),

$$
f_{r: n}(x)=\sum_{i=1}^{r} \frac{n !}{(i-1) !(n-i+1) !}\left[\left(\frac{x-1}{k}\right)^{i-1}\left(\frac{k-x+1}{k}\right)^{n-i+1}-\left(\frac{x}{k}\right)^{i-1}\left(\frac{k-x}{k}\right)^{n-i+1}\right]
$$

is obtained. Similarly, in (9) if we integrate for $r=n$,

$$
f_{n: n}(x)=\left(\frac{x}{k}\right)^{n}-\left(\frac{x-1}{k}\right)^{n}
$$

if we integrate for $r=n-1$,

$$
f_{n-1: n}(x)=n\left[\left(\frac{x}{k}\right)^{n-1}\left(\frac{k-x}{k}\right)-\left(\frac{x-1}{k}\right)^{n-1}\left(\frac{k-x+1}{k}\right)\right]+f_{n: n}(x)
$$

and if we integrate for $r=n-2$,

$$
f_{n-2: n}(x)=\frac{n(n-1)}{2 !}\left[\left(\frac{x}{k}\right)^{n-2}\left(\frac{k-x}{k}\right)^{2}-\left(\frac{x-1}{k}\right)^{n-2}\left(\frac{k-x+1}{k}\right)^{2}\right]+f_{n-1: n}(x)
$$

is obtained. Hence, we have

$$
f_{r: n}(x)=\frac{n^{\prime}}{r !(n-r) !}\left[\left(\frac{x}{k}\right)^{r}\left(\frac{k-x}{k}\right)^{n-r}-\left(\frac{x-1}{k}\right)^{r}\left(\frac{k-x+1}{k}\right)^{n-r}\right]+f_{r+1: n}(x) .
$$

Using (3.8), (3.9), (3.10) and (3.11), we obtain 


$$
f_{r: n}(x)=\sum_{i=r}^{n} \frac{n !}{i !(n-i) !}\left[\left(\frac{x}{k}\right)^{i}\left(\frac{k-x}{k}\right)^{n-i}-\left(\frac{x-1}{k}\right)^{i}\left(\frac{k-x+1}{k}\right)^{n-i}\right] .
$$

The following statement shows that (3.7) and (3.12) are equal.

$$
\sum_{i=1}^{r} \frac{n !}{(i-1) !(n-i+1) !}\left[\left(\frac{x-1}{k}\right)^{i-1}\left(\frac{k-x+1}{k}\right)^{n-i+1}-\left(\frac{x}{k}\right)^{i-1}\left(\frac{k-x}{k}\right)^{n-i+1}\right]=\sum_{i=r}^{n} \frac{n !}{i !(n-i) !}\left[\left(\frac{x}{k}\right)^{i}\left(\frac{k-x}{k}\right)^{n-i}-\left(\frac{x-1}{k}\right)^{i}\left(\frac{k-x+1}{k}\right)^{n-i}\right]
$$

Now, let us prove the correctness of the above equality with the induction method. For $r=1$, we have

$$
\left[\left(\frac{k-x+1}{k}\right)^{n}-\left(\frac{k-x}{k}\right)^{n}\right]=\sum_{i=1}^{n} \frac{n !}{i !(n-i) !}\left[\left(\frac{x}{k}\right)^{i}\left(\frac{k-x}{k}\right)^{n-i}-\left(\frac{x-1}{k}\right)^{i}\left(\frac{k-x+1}{k}\right)^{n-i}\right] .
$$

if $n=1$, then

$$
\left[\left(\frac{k-x+1}{k}\right)-\left(\frac{k-x}{k}\right)\right]=\sum_{i=1}^{1} \frac{1 !}{i !(1-i) !}\left[\left(\frac{x}{k}\right)^{i}\left(\frac{k-x}{k}\right)^{1-i}-\left(\frac{x-1}{k}\right)^{i}\left(\frac{k-x+1}{k}\right)^{1-i}\right],
$$

for $n=2$,

$$
\begin{gathered}
\frac{1}{k}=\frac{1}{k} \\
{\left[\left(\frac{k-x+1}{k}\right)^{2}-\left(\frac{k-x}{k}\right)^{2}\right]=\sum_{i=1}^{2} \frac{2 !}{i !(2-i) !}\left[\left(\frac{x}{k}\right)^{i}\left(\frac{k-x}{k}\right)^{2-i}-\left(\frac{x-1}{k}\right)^{i}\left(\frac{k-x+1}{k}\right)^{2-i}\right]} \\
\left(\frac{2 k-2 x+1}{k^{2}}\right)=\left(\frac{2 k-2 x+1}{k^{2}}\right),
\end{gathered}
$$

and for $n=3$,

$$
\begin{gathered}
{\left[\left(\frac{k-x+1}{k}\right)^{3}-\left(\frac{k-x}{k}\right)^{3}\right]=\sum_{i=1}^{3} \frac{3 !}{i !(3-i) !}\left[\left(\frac{x}{k}\right)^{i}\left(\frac{k-x}{k}\right)^{3-i}-\left(\frac{x-1}{k}\right)^{i}\left(\frac{k-x+1}{k}\right)^{3-i}\right]} \\
\left(\frac{3 k^{2}+3 x^{2}-6 k x+3 k-3 x+1}{k^{3}}\right)=\left(\frac{3 k^{2}+3 x^{2}-6 k x+3 k-3 x+1}{k^{3}}\right) .
\end{gathered}
$$

For $r=m$, let us assume that the equality holds, that is,

$$
\begin{aligned}
f_{m: n}(x) & =\sum_{i=1}^{m} \frac{n !}{(i-1) !(n-i+1) !}\left[\left(\frac{x-1}{k}\right)^{i-1}\left(\frac{k-x+1}{k}\right)^{n-i+1}-\left(\frac{x}{k}\right)^{i-1}\left(\frac{k-x}{k}\right)^{n-i+1}\right] \\
& =\sum_{i=m}^{n} \frac{n !}{i !(n-i) !}\left[\left(\frac{x}{k}\right)^{i}\left(\frac{k-x}{k}\right)^{n-i}-\left(\frac{x-1}{k}\right)^{i}\left(\frac{k-x+1}{k}\right)^{n-i}\right] .
\end{aligned}
$$

For $r=m+1$, we should show the accuracy of the equality as follows:

$$
\begin{gathered}
f_{m+1: n}(x)=\sum_{i=1}^{m+1} \frac{n !}{(i-1) !(n-i+1) !}\left[\left(\frac{x-1}{k}\right)^{i-1}\left(\frac{k-x+1}{k}\right)^{n-i+1}-\left(\frac{x}{k}\right)^{i-1}\left(\frac{k-x}{k}\right)^{n-i+1}\right] \\
=\sum_{i=m+1}^{n} \frac{n !}{i !(n-i) !}\left[\left(\frac{x}{k}\right)^{i}\left(\frac{k-x}{k}\right)^{n-i}-\left(\frac{x-1}{k}\right)^{i}\left(\frac{k-x+1}{k}\right)^{n-i}\right] .
\end{gathered}
$$

If we add the term $\frac{n !}{m !(n-m) !}\left[\left(\frac{x-1}{k}\right)^{m}\left(\frac{k-x+1}{k}\right)^{n-m}-\left(\frac{x}{k}\right)^{m}\left(\frac{k-x}{k}\right)^{n-m}\right]$ to the both sides of (3.13), 


$$
\sum_{i=1}^{m+1} \frac{n !}{(i-1) !(n-i+1) !}\left[\left(\frac{x-1}{k}\right)^{i-1}\left(\frac{k-x+1}{k}\right)^{n-i+1}-\left(\frac{x}{k}\right)^{i-1}\left(\frac{k-x}{k}\right)^{n-i+1}\right]
$$

is obtained. If we open the sum on the right side of the equation for $i=m$, then we get

$$
\begin{gathered}
\sum_{i=1}^{m+1} \frac{n !}{(i-1) !(n-i+1) !}\left[\left(\frac{x-1}{k}\right)^{i-1}\left(\frac{k-x+1}{k}\right)^{n-i+1}-\left(\frac{x}{k}\right)^{i-1}\left(\frac{k-x}{k}\right)^{n-i+1}\right] \\
=\sum_{i=m+1}^{n} \frac{n !}{i !(n-i) !}\left[\left(\frac{x}{k}\right)^{i}\left(\frac{k-x}{k}\right)^{n-i}-\left(\frac{x-1}{k}\right)^{i}\left(\frac{k-x+1}{k}\right)^{n-i}\right] \\
+\frac{n !}{m !(n-m) !}\left[\left(\frac{x-1}{k}\right)^{m}\left(\frac{k-x+1}{k}\right)^{n-m}-\left(\frac{x}{k}\right)^{m}\left(\frac{k-x}{k}\right)^{n-m}\right]-\frac{n !}{m !(n-m) !}\left[\left(\frac{x-1}{k}\right)^{m}\left(\frac{k-x+1}{k}\right)^{n-m}-\left(\frac{x}{k}\right)^{m}\left(\frac{k-x}{k}\right)^{n-m}\right] \\
=\sum_{i=m+1}^{n} \frac{n !}{i !(n-i) !}\left[\left(\frac{x}{k}\right)^{i}\left(\frac{k-x}{k}\right)^{n-i}-\left(\frac{x-1}{k}\right)^{i}\left(\frac{k-x+1}{k}\right)^{n-i}\right] .
\end{gathered}
$$

This completes the proof.

\section{REFERENCES}

[1] Khatri, C. G. (1962). Distributions of order statistics for discrete case. Annals of the Institute of Statistical Mathematics, 14(1), 167-171.

[2] Das Gupta, S. \& Sarkar, S.K. (1984). On TP2 and log-concavity. In Tong, Y.L. (ed.), Inequalities in statistics and probability, London: Institute of Mathematical, pp. 54-58.

[3] Kabe, D. G. (1969). Some distribution problems of order statistics from discrete populations. Annals of the Institute of Statistical Mathematics, 21(1), 551-556.

[4] David, H. A., \& Nagaraja, H. N. (1981). Order statistics. New York: John Willey \& Sons. David2Order Statistics1981. [5] Nagaraja, H. N. (1982). Some nondegenerate limit laws for the selection differential. Annals of Statistics, 10, 13061310 .

[6] Arnold, B. C., Balakrishnan, N., \& Nagaraja, H. N. (1992). A first course in order statistics (Vol. 54). Siam.

[7] Ahsanullah, M., \& Nevzorov, V. B. (2001). Ordered Random Variables, Nova Sci. Publ., Huntington, NY, 200(1).

[8] Çalik, S., \& Güngör, M. (2004). On the expected values of the sample maximum of order statistics from a discrete uniform distribution. Applied mathematics and computation, 157(3), 695-700.

[9] Turan, A. (2008) Kesikli Düzgün Dağılımdaki Sıra İstatistiklerin Örnek Ekstremlerinin Mometleri, Fırat University, Science Institute, Elazı̆̆.

[10] Calik, S., et al., On The Moments of Order Statistics from Discrete Distributions, Pak. J. Statist., 26 (2010), 2 , pp. 417-426.

[11] Buğatekin Turan, A.,. Generalized Moments of Sample Extremes of Order Statistics from Discrete Uniform Distribution, Firat University, Institute of Science 2011.

[12] Calik, S., \& Bugatekin, A. T. (2018). Moments of Sample Extremes of Order Statistics from Discrete Uniforms Distribution and Numerical Results. Thermal Science. 